\title{
A lower radius and mass for the transiting extrasolar planet HAT-P-8 $b^{\star}$
}

\author{
L. Mancini ${ }^{1,2}$, J. Southworth ${ }^{3}$, S. Ciceri ${ }^{1}$, J. J. Fortney ${ }^{4}$, C. V. Morley ${ }^{4}$, J. A. Dittmann ${ }^{5}$, J. Tregloan-Reed ${ }^{3}$, I. Bruni ${ }^{6}$, \\ M. Barbieri ${ }^{7}$, D. F. Evans ${ }^{3}$, G. D’ Ago ${ }^{2}$, N. Nikolov ${ }^{1}$, and Th. Henning ${ }^{1}$
}

\author{
${ }^{1}$ Max Planck Institute for Astronomy, Königstuhl 17, 69117 Heidelberg, Germany \\ e-mail: mancini@mpia.de \\ 2 Department of Physics, University of Salerno, Via Ponte Don Melillo, 84084 Fisciano (SA), Italy \\ 3 Astrophysics Group, Keele University, Staffordshire, ST5 5BG, UK \\ ${ }^{4}$ Department of Astronomy \& Astrophysics, University of California, Santa Cruz, CA 95064, USA \\ 5 Harvard-Smithsonian Center for Astrophysics, 60 Garden Street, MS 10 Cambridge, MA 02138, USA \\ 6 INAF - Osservatorio Astronomico di Bologna, Via Ranzani 1, 40127 Bologna, Italy \\ 7 INAF - Osservatorio Astronomico di Padova, Vicolo Osservatorio 5, 35122 Padova, Italy
}

Received 27 August 2012 / Accepted 15 December 2012

\begin{abstract}
Context. The extrasolar planet HAT-P-8 b was thought to be one of the more inflated transiting hot Jupiters.

Aims. By using new and existing photometric data, we computed precise estimates of the physical properties of the system.

Methods. We present photometric observations comprising eleven light curves covering six transit events, obtained using five mediumclass telescopes and telescope-defocussing technique. One transit was simultaneously obtained through four optical filters, and two transits were followed contemporaneously from two observatories. We modelled these and seven published datasets using the JKTEBOP code. The physical parameters of the system were obtained from these results and from published spectroscopic measurements. In addition, we investigated the theoretically-predicted variation of the apparent planetary radius as a function of wavelength, covering the range $330-960 \mathrm{~nm}$.

Results. We find that HAT-P-8 $\mathrm{b}$ has a significantly lower radius $\left(1.321 \pm 0.037 R_{\mathrm{Jup}}\right)$ and mass $\left(1.275 \pm 0.053 M_{\mathrm{Jup}}\right)$ compared to previous estimates $\left(1.50_{-0.06}^{+0.08} R_{\text {Jup }}\right.$ and $1.52_{-0.16}^{+0.18} M_{\text {Jup }}$ respectively). We also detect a radius variation in the optical bands that, when compared with synthetic spectra of the planet, may indicate the presence of a strong optical absorber, perhaps TiO and VO gases, near the terminator of HAT-P-8 $b$.

Conclusions. These new results imply that HAT-P- $8 \mathrm{~b}$ is not significantly inflated, and that its position in the planetary mass-radius diagram is congruent with those of many other transiting extrasolar planets.
\end{abstract}

Key words. planets and satellites: fundamental parameters - stars: fundamental parameters - stars: individual: HAT-P-8

\section{Introduction}

Transiting extrasolar planetary systems are of interest and importance, because precise measurements of their physical properties can be achieved using spectroscopic and photometric observations. Atomic and molecular absorption within the atmosphere of transiting extrasolar planets (TEPs) can also be investigated through transmission spectroscopy (e.g. Swain et al. 2008; Sing et al. 2009; Fossati et al. 2010) and simultaneous multi-colour photometry (e.g. Ballester et al. 2007; Southworth et al. 2012b; Mancini et al. 2013) of the transits. High-quality photometric observations not only enable the measurement of the masses and radii of TEPs to accuracies of a few percent (e.g. Torres et al. 2008; Southworth 2009), but also the detection of transit anomalies due to stellar pulsations (Collier Cameron et al. 2010), tidal distortion (Li et al. 2010; Leconte et al. 2011), additional bodies (moons, planets)

\footnotetext{
* Full Table 2 is only available in electronic form at the CDS via anonymous ftp to cdsarc.u-strasbg. fr (130.79.128.5) or via http://cdsarc.u-strasbg.fr/viz-bin/qcat?]/A+A/551/A11
}

(Kipping et al. 2009; Tusnski \& Valio 2011), gravity darkening (Barnes 2009; Szabó et al. 2011) and star spots (Pont et al. 2007; Rabus et al. 2009; Désert 2011). Besides the RossiterMcLaughlin effect (Queloz et al. 2000; Gaudi et al. 2007), the photometric follow-up on consecutive/close nights of transits of planets over parent-star starspots represents another fascinating method (Sanchis-Ojeda \& Winn 2011; Sanchis-Ojeda et al. 2011, 2012; Tregloan-Reed et al. 2012) to measure the skyprojected spin-orbit alignment.

The increasing number of TEPs discovered every year is progressively revealing a remarkable diversity. The improving statistical weight of this sample is useful for establishing the correct theoretical framework of planet formation and evolution. Accurate estimates of the planet properties (mass, radius, orbital semi-major axis, etc.) are vital for this purpose, and photometric follow-up of known TEPs can dramatically improve our knowledge of the planet's characteristics.

HAT-P-8 $\mathrm{b}$ is a transiting hot Jupiter found by the HATNet team (Latham et al. 2009), orbiting with a period of $~ 3.07$ days around a star of spectral type F8 (Jones \& Sleep 2000) or 
Table 1. Details of the observations presented in this work.

\begin{tabular}{|c|c|c|c|c|c|c|c|c|c|c|c|}
\hline Telescope & 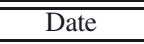 & Start/End times & 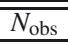 & $t_{\exp }$ & Filter & Airmass & Moon & Aperture & Scatter & PSF & $\overline{\beta \beta}$ \\
\hline Kuiper & 20091022 & $04: 03 \rightarrow 09: 10$ & 1083 & 5 & Cousins $I$ & $1.00 \rightarrow 1.9$ & $25 \%$ & $10,20,25$ & 1.54 & & \\
\hline Loiano & 20091112 & $16: 52 \rightarrow 22: 09$ & 103 & 118 & Gunn $r$ & $1.02 \rightarrow 1.01 \rightarrow 1.55$ & $23 \%$ & $25,45,60$ & 1.15 & 1963 & 1.4 \\
\hline INT & 20100828 & $20: 44 \rightarrow 04: 02$ & 131 & 140 & Strömgren $y$ & $2.04 \rightarrow 1.00 \rightarrow 1.18$ & $84 \%$ & $45,65,90$ & 0.47 & 3217 & 1.7 \\
\hline Loiano & 20111005 & $18: 01 \rightarrow 02: 29$ & 469 & 50 & Gunn $i$ & $1.12 \rightarrow 1.01 \rightarrow 1.45$ & $61 \%$ & $19,40,60$ & 1.15 & 908 & 1.4 \\
\hline CA $1.23 \mathrm{~m}$ & 20111005 & $19: 27 \rightarrow 03: 07$ & 166 & 70 & Johnson $R$ & $1.08 \rightarrow 1.05 \rightarrow 1.54$ & $61 \%$ & $26,38,55$ & 0.83 & 1662 & 1.0 \\
\hline $\mathrm{CA} 2.2 \mathrm{~m}$ & 20120826 & $20: 31 \rightarrow 04: 48$ & 160 & 80 & sdss $u$ & $1.52 \rightarrow 1.00 \rightarrow 1.55$ & $77 \%$ & $25,55,75$ & 2.37 & 2827 & 1.0 \\
\hline $\mathrm{CA} 2.2 \mathrm{~m}$ & 20120826 & $20: 31 \rightarrow 04: 48$ & 157 & 80 & Gunn $g$ & $1.52 \rightarrow 1.00 \rightarrow 1.55$ & $77 \%$ & 70 & 0.97 & 2463 & 1.2 \\
\hline CA $2.2 \mathrm{~m}$ & 20120826 & $20: 31 \rightarrow 04: 48$ & 159 & 80 & Gunn $r$ & $1.52 \rightarrow 1.00 \rightarrow 1.55$ & $77 \%$ & $30,60,90$ & 0.71 & 2642 & 1.5 \\
\hline $\mathrm{CA} 2.2 \mathrm{~m}$ & 20120826 & $20: 31 \rightarrow 04: 48$ & 162 & 80 & Gunn $z$ & $1.52 \rightarrow 1.00 \rightarrow 1.55$ & $77 \%$ & $28,55,80$ & 0.92 & 1662 & 1.1 \\
\hline Loiano & 20121002 & $17: 45 \rightarrow 02: 32$ & 232 & 70 & Johnson $I$ & $1.03 \rightarrow 1.00 \rightarrow 1.92$ & $92 \%$ & $23,40,65$ & 0.79 & 2521 & 1.0 \\
\hline CA $1.23 \mathrm{~m}$ & 20121002 & $20: 07 \rightarrow 03: 35$ & 373 & 50 & Johnson $I$ & $1.11 \rightarrow 1.00 \rightarrow 2.16$ & $92 \%$ & $35,60,90$ & 1.69 & 2290 & 1.8 \\
\hline
\end{tabular}

Notes. $N_{\text {obs }}$ is the number of observations, Moon is the fractional illumination of the Moon at the midpoint of the transit, and $t_{\exp }$ is the exposure time in seconds. The aperture sizes are the radii in pixels of the software apertures for the star, inner sky and outer sky, respectively. Scatter is the rms scatter of the data versus a fitted model, in mmag. Target mean PSF area in $\mathrm{px}^{2}$ is also reported for each dataset. Times and dates are in UT. $\beta$ is the ratio between the noise levels due to Poisson noise and to combined Poisson and red noise.

F5 (Bergfors et al. 2012). At the time of its discovery it was labelled as one of the most inflated transiting giant planets, with a measured mass and radius of $M_{\mathrm{b}}=1.52_{-0.16}^{+0.18} M_{\mathrm{Jup}}$ and $R_{\mathrm{b}}=1.50_{-0.06}^{+0.08} R_{\text {Jup }}$, respectively. These values differ by $2-3 \sigma$ from the theoretical predictions of Fortney et al. (2007).

The Rossiter-McLaughlin effect has been detected in the HAT-P-8 system using radial velocity observations from the SOPHIE and FIES spectrographs. Simpson et al. (2011) found a sky-projected orbital obliquity of $\lambda=-9.7_{-7.7}^{+9.0}$ degrees and Moutou et al. (2011) found $\lambda=-17_{-11.5}^{+9.2}$ degrees; both values are consistent with alignment between the orbital axis of the planet and the rotational axis of the star. The two studies between them suggested lower values for $M_{\mathrm{b}}$ and $R_{\mathrm{b}}$, but neither calculated the physical properties of the system.

Bergfors et al. (2012) have found a faint companion to the HAT-P-8 system using lucky imaging observations with the AstraLux Norte instrument at the Calar Alto $2.2 \mathrm{~m}$ telescope. The companion, a likely M2-4 dwarf, is at an angular distance of $1.027 \pm 0.011$ arcsec and is fainter in the SDSS $i^{\prime}$ and $z^{\prime}$ passbands by $\Delta i^{\prime}=7.34 \pm 0.10 \mathrm{mag}$ and $\Delta z^{\prime}=6.68 \pm 0.07 \mathrm{mag}$. The faintness of this star means that it has a negligible effect on optical observations of HAT-P-8.

In this work we present eleven new follow-up light curves covering six transits in the HAT-P-8 system, obtained using five $1.2-2.5 \mathrm{~m}$ telescopes. We augment these data with previously published observations of seven transits, and measure the physical properties of the system. We find a substantially lower mass and radius for the planet, removing its outlier status and relegating it to a more well-populated part of the planetary mass-radius diagram.

Our paper is structured as follows. In Sect. 2 we describe the observations and data reduction. In Sect. 3 we analyse the data, and in Sect. 4 we obtain refined orbital ephemerides and physical properties of the HAT-P-8 system. In Sect. 5 we investigate the variation of the planetary radius as function of wavelength. Several anomalies detected in the light curves are discussed in Sect. 6, whereas in Sect. 7 we summarize the results and draw our conclusions.

\section{Observations and data reduction}

Six transits of HAT-P- $8 \mathrm{~b}$ were monitored by five different telescopes between 2009 and 2012. Two transits were followed simultaneously by two of the telescopes, and one was simultaneously observed through four optical filters. Except the first,
Table 2. Excerpts of the light curves of HAT-P-8.

\begin{tabular}{lcccc}
\hline \hline Telescope & Filter & BJD (TDB) & Diff. mag. & Uncertainty \\
\hline Loiano & $r$ & 2455148.210781 & 0.0009980 & 0.0018456 \\
Loiano & $r$ & 2455148.214867 & 0.0000050 & 0.0017916 \\
Loiano & $i$ & 2455840.256418 & 0.0000785 & 0.0016988 \\
Loiano & $i$ & 2455840.258316 & 0.0006256 & 0.0016925 \\
CAHA & $R$ & 2455840.319832 & 0.0007127 & 0.0013972 \\
CAHA & $R$ & 2455840.329288 & -0.0019752 & 0.0013798 \\
INT & $y$ & 2455437.412356 & 0.0052976 & 0.0009779 \\
INT & $y$ & 2455437.414575 & 0.0056092 & 0.0009998 \\
Kuiper & $I$ & 2455126.66906 & -0.99507 & - \\
Kuiper & $I$ & 2455126.66930 & -0.99959 & - \\
\hline
\end{tabular}

Notes. This table will be made available at the CDS. A portion is shown here for guidance regarding its form and content.

all our transit observations were performed with the telescope defocussing method, in order to minimise the effect of Poisson, scintillation and flat-fielding noise (Southworth et al. 2009). We used autoguiding during all the observations, and the stars incurred a drift of 10 pixels or less over each observing sequence. The corresponding night logs are reported in Table 1, and the differential photometry is tabulated in Table 2 and plotted in Figs. 1 and 2 .

\subsection{Kuiper $1.55 m$ telescope}

We observed one transit of HAT-P-8 in October 2009 using the University of Arizona's 1.55 m Kuiper telescope on Mt. Bigelow, Arizona. We used the Mont $4 \mathrm{k}$ CCD, binned $3 \times 3$ to $0.43^{\prime \prime} /$ pixel, for a total field of view (FOV) of $9.7^{\prime} \times 9.7^{\prime}$, and an Arizona- $I$ filter ${ }^{1}$. The observations were conducted with autoguiding and the telescope focussed. Due to the bright nature of the star we used $5 \mathrm{~s}$ exposure times, which resulted in an observing cadence of $\approx 30 \mathrm{~s}$.

Systematic effects were minimised by autoguiding, keeping star wander to less than 5 pixels $\left(2.15^{\prime \prime}\right)$ over the course of the night. The resulting images were bias-subtracted, flat-fielded, and bad pixel-cleaned in the usual manner. Aperture photometry was performed using an IDL $^{2}$ pipeline utilising the FIND and

1 The transmission curve for this filter is shown at:

james . as . arizona . edu/ \{psmith\}/61inch/FILTERS/harris.jpg

2 The acronym IDL stands for Interactive Data Language and is a trademark of ITT Visual Information Solutions. For further details see http: //www . ittvis . com/ProductServices/IDL . aspx 

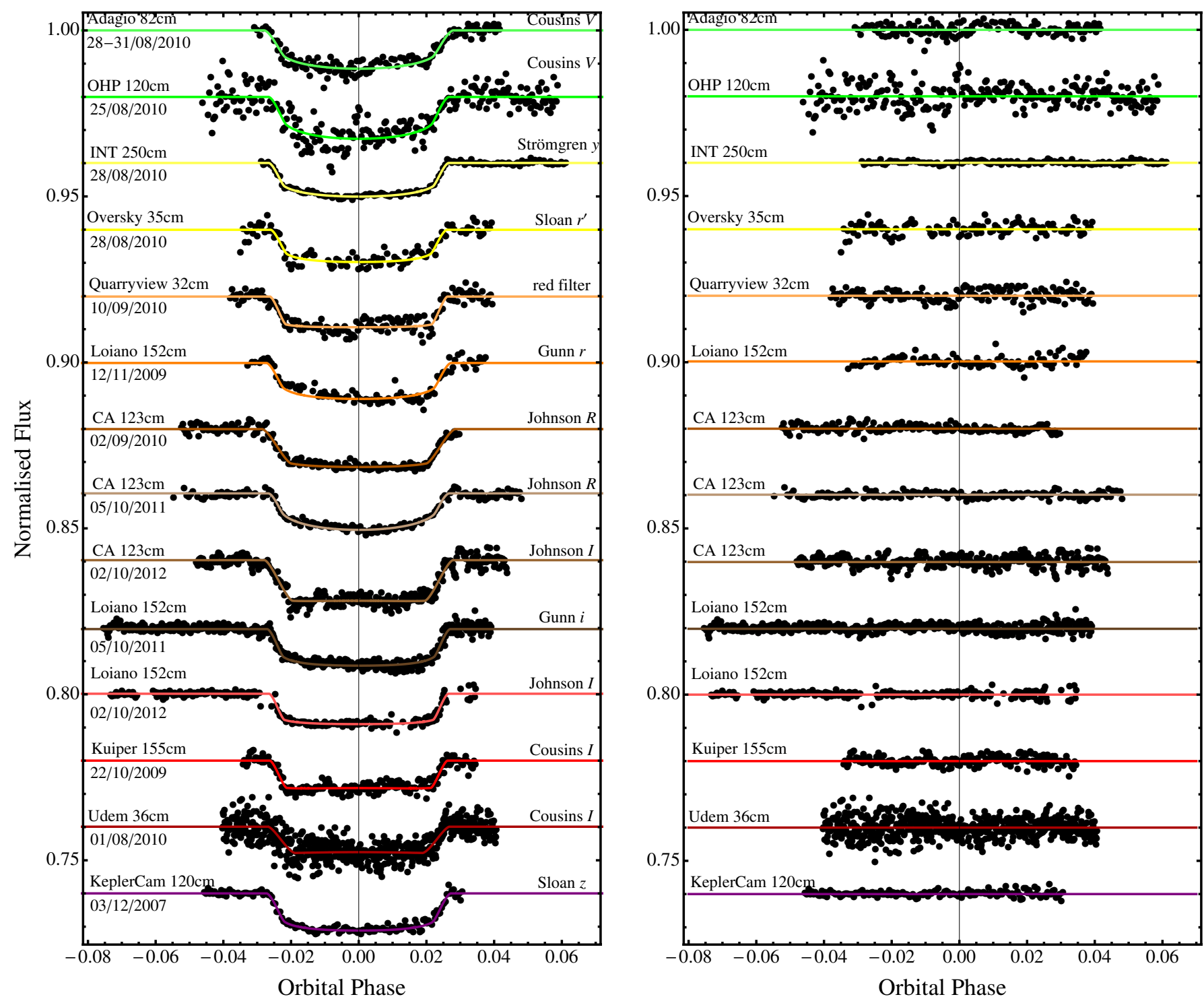

Fig. 1. Phased light curves of HAT-P-8 compared to the best JKTEBOP fits using the quadratic LD law (left-hand panel). They are ordered according to central wavelength of the filter used. The residuals of the fits are plotted in the right-hand panel, offset to bring them into the same relative position as the corresponding best fit in the left-hand panel.

APER (Stetson 1987) tasks available in the NASA Astronomy User's Library ${ }^{3}$. The size of the aperture was chosen to minimise scatter in the data and was 10 pixels $\left(4.3^{\prime \prime}\right)$ in radius. Several combinations of reference stars were considered, and the one which gave the lowest scatter in the final light curve was adopted. The 1083 original datapoints were binned to yield 216 final datapoints.

\subsection{Cassini $1.52 m$ telescope}

One transit of HAT-P-8 was observed in November 2009, two in October 2011 and one in October 2012, using the $1.52 \mathrm{~m}$ Cassini Telescope at the Astronomical Observatory of Bologna in Loiano (Italy). We have previously used this telescope several times to observe planetary transits (e.g. Southworth et al. 2012a), with the BFOSC (Bologna Faint

\footnotetext{
3 The IDL Astronomy User's Library (ASTROLIB) is available at http://idlastro.gsfc.nasa.gov/
}

Object Spectrograph and Camera) instrument operated in imaging mode.

The CCD was used unbinned, giving a plate scale of $0.58^{\prime \prime} /$ pixel, for a total FOV of $13^{\prime} \times 12.6^{\prime}$, and the telescope was autoguided and defocussed. The first transit was observed through a Gunn $r$ filter, the two 2011 transits through a Gunn $i$ filter, and the last one through a Johnson $I$ filter. The first 2011 transit suffered from systematic noise due to a bad pixel in the aperture of the target star, so we did not use these data in our analysis. The transit of 2012 was disturbed by clouds, which affected the photometry particularly at the end of the transit. We removed the points compromised by the clouds.

The observations were analysed using the IDL pipeline from Southworth et al. (2009). The images were debiased and flatfielded using standard methods, then subjected to aperture photometry using the APER task. Pointing variations were followed by cross-correlating each image against a reference image. We chose the aperture sizes and comparison stars which yielded the lowest scatter in the final differential-photometry light curve. 
The relative weights of the comparison stars were optimised simultaneously with fitting a second-order polynomial to the outside-transit observations in order to normalise them to unit flux.

\subsection{Isaac Newton Telescope}

One transit of HAT-P-8 was monitored using the $2.5 \mathrm{~m}$ Isaac Newton Telescope (INT), La Palma (Spain), equipped with the Wide Field Camera (WFC) at prime focus. We used only one of the four CCDs, unbinned and with a plate scale of $0.33^{\prime \prime} /$ pixel, for a total FOV of $12.6^{\prime} \times 11.3^{\prime}$. The telescope was defocussed and autoguided, and the observations were obtained through a Strömgren $y$ filter. A few data points at the start of the observing sequence were rejected as they are affected by systematic noise (due probably to high airmass $>2$ ). The observations were reduced in the same way as those from the Cassini Telescope (Sect. 2.2).

\subsection{Calar Alto $1.23 \mathrm{~m}$ telescope}

Three transits of HAT-P-8 b, two in October 2011 and one in October 2012, were obtained using the $1.23 \mathrm{~m}$ telescope at the German-Spanish Calar Alto Observatory (CAHA) near Almería, Spain. Autoguiding was used. During the 2011 observations, we used the $2 \mathrm{k} \times 2 \mathrm{k}$ SITE\# $2 \mathrm{~b}$ optical CCD, which had ${ }^{4}$ a FOV of $16^{\prime} \times 16^{\prime}$ and a pixel scale of $0.5^{\prime \prime}$ per pixel. We defocussed the telescope, read out only a small window in order to limit the dead time between exposures, and observed through a Johnson $R$ filter. The first transit was incomplete due to clouds, so was not included in our analysis. The 2012 transit was obtained through a Johnson $I$ filter with the new DLR-MKIII camera, which is equipped with an e2v CCD231-84-NIMO-BI-DD sensor with $4 \mathrm{k} \times 4 \mathrm{k}$ pixels and a FOV of $21^{\prime} \times 21^{\prime}$ at $0.3^{\prime \prime}$ per pixel. Unfortunately, the operator did not included appropriate reference stars in the FOV, and the scatter of the resulting light curve is higher than those of 2011. All the observations were reduced as for the Cassini Telescope (Sect. 2.2).

\subsection{Calar Alto $2.2 m$ telescope}

We observed one full transit of HAT-P-8 on the night of 2012 August 26, using the $2.2 \mathrm{~m}$ telescope and BUSCA imager at CAHA. BUSCA is designed for simultaneous four-colour photometry: the light is split into four wavelength bands from UV to visual IR using three dichroics. In the four corresponding focal planes the same area of the sky is imaged onto $4 \mathrm{k} \times 4 \mathrm{k} 15 \mu \mathrm{m}$ pixel CCDs. For our observations we chose to have the SDSS $u$ filter in the bluest arm and standard Calar Alto Gunn $g, r$ and $z$ filters in the other three arms. This choice led to a reduced FOV (from $12^{\prime} \times 12^{\prime}$ to a circle of $6^{\prime}$ in diameter), but had the advantage of a much better throughput in $g r z$ compared to the default Strömgren filters. We defocussed BUSCA in such a way as to have as much signal as possible in the $u$ band whilst remaining in the linear regime in the other passbands. The CCDs were binned $2 \times 2$ to shorten the readout time. The autoguider was operated in-focus. The observations were reduced in the same way as those from the Cassini Telescope (Sect. 2.2) and the resulting light curves are plotted in Fig. 2.

\footnotetext{
4 This CCD has been decommissioned.
}

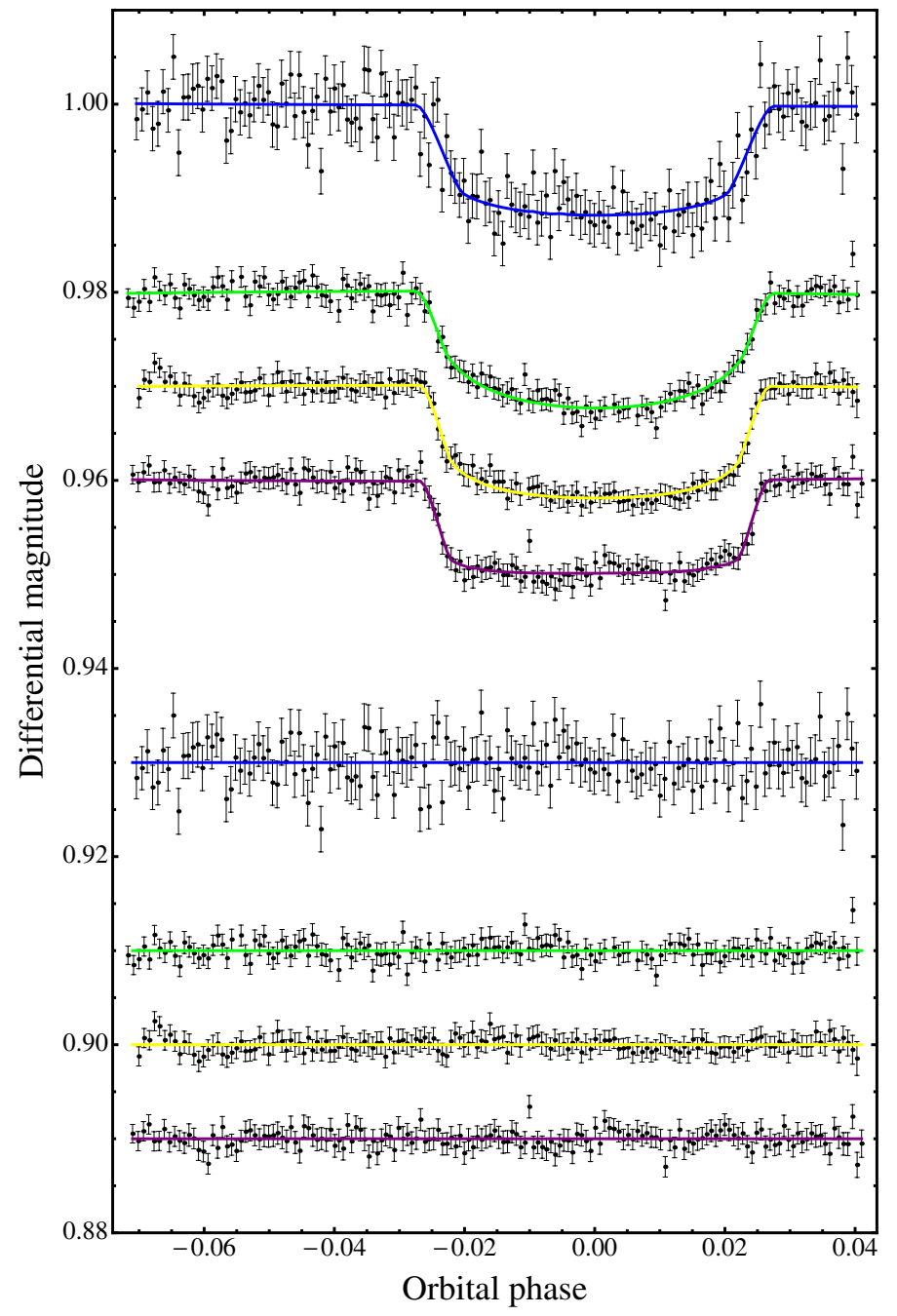

Fig. 2. Phased BUSCA light curves of HAT-P-8 compared to the best JKTEBOP fits using the quadratic LD law. They are ordered according to central wavelength of the filter used (sdss $u$, Gunn $g$, Gunn $r$, Gunn $z$ ). The residuals of the fits are plotted at the base of the figure, offset from zero.

\section{Light curve analysis}

Section 2 introduced eleven transit light curves which were suitable for detailed analysis. These were each fitted using the JKTEBOP $^{5}$ code (Southworth et al. 2004), which represents the star and planet as biaxial spheroids. The primary parameters of the fit were the orbital inclination, $i$, the sum and ratio of the fractional radii of the star and planet, $r_{\mathrm{A}}+r_{\mathrm{b}}$ and $k=r_{\mathrm{b}} / r_{\mathrm{A}}$, defined as $r_{\mathrm{A}}=R_{\mathrm{A}} / a$ and $r_{\mathrm{b}}=R_{\mathrm{b}} / a$, and transit midpoint, $T_{0}$. The orbital semimajor axis is $a$ and, $R_{\mathrm{A}}$ and $R_{\mathrm{b}}$ are the absolute radii of the two celestial bodies.

Once a fit was available for each dataset, we rescaled the errorbars to give a reduced $\chi^{2}$ of $\chi_{v}^{2}=1$. This step is necessary because the APER aperture photometry procedure has a tendency to underestimate the measurement errors. Then, in order to take systematic noise into account, we inflated the errorbars further using the $\beta$ approach (e.g. Gillon et al. 2006; Winn et al. 2008, 2009; Gibson et al. 2008; Nikolov et al. 2012). We calculated $\beta$ values for between two and ten datapoints for each light curve, and adopted the largest $\beta$ value.

\footnotetext{
5 JKTEBOP is written in FORTRAN77 and the source code is available at http://www . astro.keele.ac.uk/ jkt/
} 
Table 3. Parameters of the JKTEBOP fits to the light curves of HAT-P-8.

\begin{tabular}{|c|c|c|c|c|c|c|}
\hline Source & Filter & $r_{\mathrm{A}}+r_{\mathrm{b}}$ & $k$ & $i^{\circ}$ & $r_{\mathrm{A}}$ & $r_{\mathrm{b}}$ \\
\hline Loiano \#1 & Gunn $r$ & $0.177 \pm 0.013$ & $0.0921 \pm 0.0034$ & $87.3 \pm 2.3$ & $0.162 \pm 0.012$ & $0.0149 \pm 0.0014$ \\
\hline Loiano \#2 & Gunn $i$ & $0.1695 \pm 0.0030$ & $0.0943 \pm 0.0022$ & $89.2 \pm 1.0$ & $0.1460 \pm 0.0026$ & $0.01460 \pm 0.00046$ \\
\hline Loiano \#3 & Johnson $I$ & $0.1674 \pm 0.0035$ & $0.0884 \pm 0.0010$ & $88.5 \pm 1.0$ & $0.1538 \pm 0.0032$ & $0.01360 \pm 0.00034$ \\
\hline CA $1.23 \mathrm{~m} \mathrm{\# 1}$ & Johnson $R$ & $0.218 \pm 0.012$ & $0.1006 \pm 0.0021$ & $82.7 \pm 1.1$ & $0.198 \pm 0.010$ & $0.0199 \pm 0.0012$ \\
\hline CA $1.23 \mathrm{~m} \mathrm{\# 2}$ & Johnson $R$ & $0.1685 \pm 0.0061$ & $0.0888 \pm 0.0032$ & $88.7 \pm 1.6$ & $0.1548 \pm 0.0058$ & $0.01374 \pm 0.00084$ \\
\hline CA $1.23 \mathrm{~m} \mathrm{\# 3}$ & Johnson $I$ & $0.208 \pm 0.018$ & $0.1056 \pm 0.0042$ & $83.3 \pm 1.6$ & $0.188 \pm 0.016$ & $0.0199 \pm 0.0018$ \\
\hline CA $2.2 \mathrm{~m}$ & $\operatorname{sdss} u$ & $0.199 \pm 0.024$ & $0.0993 \pm 0.0039$ & $84.1 \pm 2.5$ & $0.181 \pm 0.021$ & $0.0180 \pm 0.0026$ \\
\hline $\mathrm{CA} 2.2 \mathrm{~m}$ & Gunn $g$ & $0.1720 \pm 0.0054$ & $0.0926 \pm 0.0024$ & $89.9 \pm 1.4$ & $0.157 \pm 0.050$ & $0.01458 \pm 0.00057$ \\
\hline $\mathrm{CA} 2.2 \mathrm{~m}$ & Gunn $r$ & $0.1692 \pm 0.0051$ & $0.0959 \pm 0.0018$ & $88.7 \pm 1.4$ & $0.1544 \pm 0.0046$ & $0.01480 \pm 0.00059$ \\
\hline CA $2.2 \mathrm{~m}$ & Gunn $z$ & $0.1695 \pm 0.0059$ & $0.0917 \pm 0.0016$ & $88.3 \pm 1.5$ & $0.1552 \pm 0.0053$ & $0.01424 \pm 0.00058$ \\
\hline INT & Strömgren $y$ & $0.1699 \pm 0.0052$ & $0.0886 \pm 0.0016$ & $87.9 \pm 1.2$ & $0.1560 \pm 0.0047$ & $0.01383 \pm 0.00056$ \\
\hline Kuiper & Cousins $I$ & $0.175 \pm 0.012$ & $0.0871 \pm 0.0026$ & $86.4 \pm 1.6$ & $0.161 \pm 0.012$ & $0.0140 \pm 0.0010$ \\
\hline UDEM & Cousins $I$ & $0.220 \pm 0.032$ & $0.0836 \pm 0.0039$ & $81.7 \pm 2.6$ & $0.203 \pm 0.030$ & $0.0170 \pm 0.0021$ \\
\hline Adagio & Cousins $V$ & $0.192 \pm 0.017$ & $0.0956 \pm 0.0034$ & $85.3 \pm 2.1$ & $0.175 \pm 0.015$ & $0.0167 \pm 0.0018$ \\
\hline $\mathrm{OHP}$ & Cousins $V$ & $0.172 \pm 0.031$ & $0.0977 \pm 0.0088$ & $87.4 \pm 3.9$ & $0.157 \pm 0.027$ & $0.0153 \pm 0.0031$ \\
\hline Quarryview & Red filter & $0.169 \pm 0.011$ & $0.0901 \pm 0.0024$ & $87.5 \pm 2.1$ & $0.155 \pm 0.010$ & $0.0140 \pm 0.0011$ \\
\hline Oversky & Sloan $r^{\prime}$ & $0.171 \pm 0.016$ & $0.0888 \pm 0.0033$ & $86.9 \pm 2.6$ & $0.157 \pm 0.015$ & $0.0140 \pm 0.0016$ \\
\hline KeplerCam & Sloan $z$ & $0.1781 \pm 0.0081$ & $0.0955 \pm 0.0013$ & $86.9 \pm 1.3$ & $0.1626 \pm 0.0073$ & $0.01553 \pm 0.00084$ \\
\hline Final results & & & $0.09208 \pm 0.00049$ & $87.08 \pm 0.36$ & $0.1590 \pm 0.0014$ & $0.01468 \pm 0.00017$ \\
\hline Latham et al. (2009) & & $0.1725_{-0.00048}^{+0.00094}$ & $0.0953 \pm 0.0009$ & $87.5_{-0.9}^{+1.9}$ & $0.1575_{-0.0042}^{+0.0084}$ & $0.01501_{-0.00054}^{+0.00095}$ \\
\hline Simpson et al. (2011) & & $0.1783_{-0.00063}^{+0.00060}$ & $0.09135 \pm 0.00089$ & $87.80_{-0.77}^{+0.75}$ & $0.1634_{-0.0056}^{+0.0053}$ & $0.01493_{-0.00066}^{+0.00063}$ \\
\hline
\end{tabular}

Notes. The final parameters, given in bold, are the weighted means of the results for the 11 datasets. Results from the literature are included at the base of the table for comparison.

Limb darkening (LD) was accounted for using a quadratic law. The linear LD coefficient was fitted, whereas the non-linear one was fixed at a theoretically predicted value (Claret 2004), but perturbed by \pm 0.1 during the process of error estimating. The atmospheric parameters of the star assumed for deriving the limb-darkening coefficients were: $T_{\text {eff }}=6130, \log g=4.15$, $[\mathrm{Fe} / \mathrm{H}]=+0.01, V_{\text {micro }}=2 \mathrm{~km} \mathrm{~s}^{-1}$. Uncertainties in the fitted parameters from each solution were calculated in two ways: from 1000 Monte Carlo simulations and with a residual-permutation algorithm (see Southworth 2008). The larger of the two possible error bars was retained in each case. The light curves and their best-fitting models are shown in Figs. 1 and 2, whereas the parameters of each fit are reported in Table 3.

We also attempted to fit all light curves simultaneously using the TAP Gazak et al. (2012) code, but were unsuccessful. Analyses of individual light curves, however, yielded similar results and uncertainties as to those from JKTEBOP.

\subsection{Datasets taken from literature}

Latham et al. (2009) reported three z-band transits (1451 points in total), only one of which was observed in its entirety, obtained with the $1.2 \mathrm{~m}$ telescope and KeplerCam at the F. L. Whipple Observatory, US. We converted the timestamps into orbital phase (using their ephemeris), sorted them and binned them into 154 points.

Moutou et al. (2011) presented five datasets, two of them obtained by the Adagio Association with an $82 \mathrm{~cm}$ telescope ( $V$ band; 720 points), one from the $1.20 \mathrm{~m}$ telescope at Observatoire de Haute-Provence ( $V$ band; 256 points), one from a $35 \mathrm{~cm}$ telescope at the Oversky observatory ( $R$ band; 569 points binned in 113 points), and one from a $32 \mathrm{~cm}$ telescope at the Quarryview Observatory ( $R$ band, 157 points). The two Adagio datasets were phased-binned into 144 points before analysis, to save computing time. The Oversky data were also binned, into 113 points.

One transit was observed with the $36 \mathrm{~cm}$ Universidad de Monterrey Observatory (UDEM) telescope ( $I$ band; 639 points).
These data were reported by Todorov et al. (2012). Finally, one very good light curve was obtained by Hormuth using the CAHA $1.23 \mathrm{~m}$ telescope ( $R$ band; 176 points). These data are available from TRESCA web site and were already studied by Simpson et al. (2011). All of the light curves in this section were fitted using JKTEBOP in the same manner as our own data.

\subsection{Final photometric parameters}

All the light curves we fitted are shown in Figs. 1 and 2. The parameters of the fits are given in Table 3 . In order to calculate the final photometric parameters we took the weighted mean of the individual values and uncertainties. This process highlighted the poor agreement between the different datasets. The $\chi_{v}^{2}$ of the parameters of the individual light curves with respect to the final weighted means are 1.5 for $r_{\mathrm{A}}, 2.4$ for $i$ and 2.7 for $r_{\mathrm{b}}$. The worst agreement is found for $k$, where $\chi_{v}^{2}=6.9$. Analogous situations have been found many times in the course of the Homogeneous Studies project (Southworth 2008, 2009, 2010, 2011, 2012), but the agreement is rarely this poor.

Inspection of Fig. 1 gives a clue to this problem. The residuals of the fits to almost all of the available light curves, including our own, exhibit systematic deviations from zero. This correlated noise means the amount of information available in the observational data is lower than suggested by the number of datapoints and their uncertainties. We have already increased our errorbars due to correlated noise using the $\beta$ approach (Sect. 3), but this was not enough to solve the problem. Fortunately, we have 18 separate light curves which have been analysed independently. Making the assumption that correlated noise in data taken on different nights using different telescopes is independent, we can account for it by inflating the errorbars on the final photometric parameters by $\sqrt{\chi_{v}^{2}}$ to give $\chi_{v}^{2}=1.0$ for each parameter. Because our results are based on 18 different datasets, we are confident that our analysis has yielded reliable parameters. The light curve anomalies are discussed further in Sect. 5. 
(a)

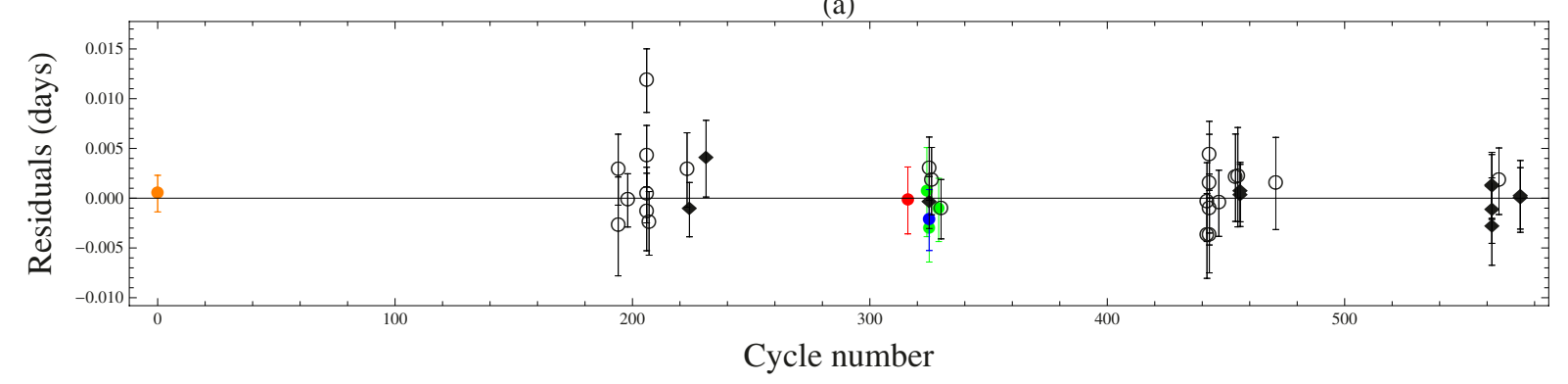

(b)

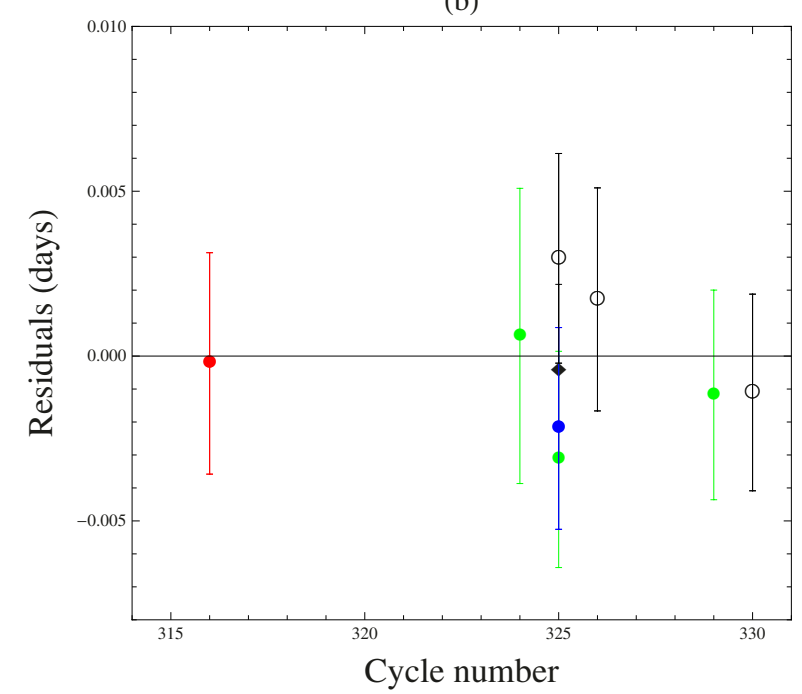

(c)

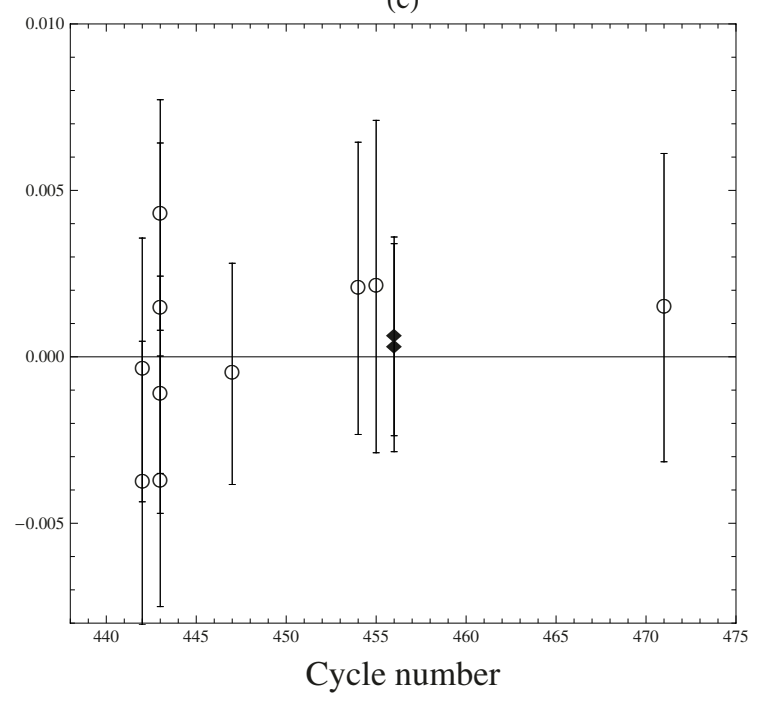

Fig. 3. Panel a) plot of the residuals of the timings of mid-transit of HAT-P-8 b versus a linear ephemeris. The timings in black are from this work, orange from Latham et al. (2009), green from Moutou et al. (2011), red from Todorov et al. (2012), and blue from Hormuth (TRESCA). The other timings obtained by amateur astronomers are plotted using open circles. Panels b) and c) are zooms in to the two best sampled regions.

\subsection{Orbital period determination}

The transit time for each dataset was obtained using JKTEBOP, and uncertainties were estimated by Monte Carlo simulations. In the determination of the orbital period of the HAT-P-8 system, we also considered 23 timings measured by amateur astronomers and available on the ETD $^{6}$ website (see Table 4).

We excluded from the analysis the incomplete ETD light curves and those with a Data Quality index higher than 3. All timings were placed on BJD(TDB) time system. The resulting measurements of transit midpoints were fitted with a straight line to obtain a new orbital ephemeris:

$$
T_{0}=\mathrm{BJD}(\mathrm{TDB}) 2454437.6742(14)+3.0763458(24) E,
$$

where $E$ is the number of orbital cycles after the reference epoch (which we take to be the midpoint of the first transit observed by Latham et al. 2009) and quantities in brackets denote the uncertainty in the final digit of the preceding number. The fit has $\chi_{v}^{2}=5.30$, and the uncertainties given above have been increased to account for this. The large $\chi_{v}^{2}$ indicates that the uncertainties in the various $T_{0}$ measurements are too small, most probably due to the systematic differences between the light curves and their best fits as noted in Sect. 3.2. A plot of the residuals around the fit is shown in Fig. 3 and does not indicate any clear systematic deviation from the predicted transit times. We therefore take the conservative option of not interpreting the large $\chi_{v}^{2}$ as a suggestion of transit timing variations.

\footnotetext{
6 The Exoplanet Transit Database (ETD) website can be found at http://var2 . astro.cz/ETD
}

\section{The physical properties of HAT-P-8}

Following Southworth (2009), we estimated the physical properties of the HAT-P-8 system from the photometric parameters measured in Sect. 3, published spectroscopic results, and theoretical stellar models. The orbital eccentricity, velocity amplitude and metallicity of the star, $\left(e=0, K_{\mathrm{A}}=153.1 \pm 3.9\right.$, $[\mathrm{Fe} / \mathrm{H}]=+0.01 \pm 0.08)$ were taken from Latham et al. (2009), and for the parent-star effective temperature $\left(T_{\text {eff }}=6130 \pm 80\right)$ we used that measured by Knutson et al. (2010).

An initial estimate of the velocity amplitude of the planet $\left(K_{\mathrm{b}}\right)$ was iteratively refined by calculating the system properties using standard formulae (e.g. Hilditch 2001), and comparing the observed $T_{\text {eff }}$ and $r_{\mathrm{A}}$ with values of $T_{\text {eff }}$ and $R_{\mathrm{A}} / a$ predicted by theoretical models for the calculated mass of the star. This calculation was performed over a grid of ages and for five different sets of stellar models (see Southworth 2010). Statistical errors were propagated by a perturbation analysis. The resulting estimates of the physical properties are given in Table 5. The final set of physical properties was obtained by taking the unweighted mean of the five sets of values found from the different stellar models, which also allowed us to obtain an estimate of the systematic errors inherent in the use of stellar theory. The results of this process are given in Table 6.

Finally, following the method delineated by Enoch et al. (2010) and improved by Southworth (2011), we used empirical measurements of stars in detached eclipsing binary (dEB) systems to calibrate the parent star of the HAT-P- 8 system. This allowed us to measure the physical properties of the system 
Table 4. Transit mid-times of HAT-P-8 and their residuals.

\begin{tabular}{|c|c|c|c|}
\hline $\begin{array}{l}\text { Time of minimum } \\
\text { BJD(TDB) }-2400000\end{array}$ & $\begin{array}{c}\text { Cycle } \\
\text { no. }\end{array}$ & $\begin{array}{c}\text { Residual } \\
\text { (JD) }\end{array}$ & Reference \\
\hline $54437.67461 \pm 0.00044$ & 0 & 0.00046 & Latham et al. (2009) \\
\hline $55034.4824 \pm 0.0031$ & 194 & -0.00284 & Srdoc (AXA) \\
\hline $55034.4881 \pm 0.0017$ & 194 & 0.00286 & Kučáková H. (TRESCA) \\
\hline $55046.7904 \pm 0.0008$ & 198 & -0.00022 & Norby (AXA) \\
\hline $55071.400 \pm 0.002$ & 206 & -0.00138 & Ayiomamitis (AXA) \\
\hline $55071.4017 \pm 0.0009$ & 206 & -0.00032 & Srdoc (AXA) \\
\hline $55071.4056 \pm 0.0012$ & 206 & 0.00422 & Kučáková H. (TRESCA) \\
\hline $55071.4132 \pm 0.0013$ & 206 & 0.01182 & Trnka J. (TRESCA) \\
\hline $55074.4752 \pm 0.0013$ & 207 & -0.00253 & Srdoc (AXA) \\
\hline $55123.7021 \pm 0.0018$ & 223 & 0.00284 & Tieman B. (TRESCA) \\
\hline $55126.77446 \pm 0.00079$ & 224 & -0.00115 & This work (Kuiper $155 \mathrm{~cm}$ ) \\
\hline $55148.3140 \pm 0.0019$ & 231 & 0.00397 & This work (Loiano $152 \mathrm{~cm}$ ) \\
\hline $55409.7992 \pm 0.0012$ & 316 & -0.00022 & Todorov et al. (2012) (Udem $36 \mathrm{~cm}$ ) \\
\hline $55434.4108 \pm 0.0023$ & 324 & 0.00061 & Moutou et al. (2011) (OHP $120 \mathrm{~cm})$ \\
\hline $55437.48610 \pm 0.00043$ & 325 & -0.00043 & This work (INT $250 \mathrm{~cm}$ ) \\
\hline $55437.4834 \pm 0.0011$ & 325 & -0.00313 & Moutou et al. (2011) (Oversky $35 \mathrm{~cm})$ \\
\hline $55437.48434 \pm 0.00088$ & 325 & -0.00219 & Hormuth F. (CA $123 \mathrm{~cm})$ \\
\hline $55437.4895 \pm 0.0010$ & 325 & 0.00297 & Ruocco N. (TRESCA) \\
\hline $55440.5646 \pm 0.0012$ & 326 & 0.00171 & Marino G. (TRESCA) \\
\hline $55449.79074 \pm 0.00099$ & 329 & -0.00118 & Moutou et al. (2011) (Hose $32 \mathrm{~cm}$ ) \\
\hline $55452.86716 \pm 0.00079$ & 330 & -0.00110 & Hose K. (TRESCA) \\
\hline $55797.4152 \pm 0.0018$ & 442 & -0.00379 & Dřevěný R., Kuchtak B. (TRESCA) \\
\hline $55797.4186 \pm 0.0015$ & 442 & -0.00039 & Brát L. (TRESCA) \\
\hline $55800.4916 \pm 0.0013$ & 443 & -0.00374 & Brát L. (TRESCA) \\
\hline $55800.4942 \pm 0.0011$ & 443 & -0.00114 & Trnka J. (TRESCA) \\
\hline $55800.4968 \pm 0.0025$ & 443 & 0.00146 & Zibar M. (TRESCA) \\
\hline $55800.4996 \pm 0.0010$ & 443 & 0.00426 & Marek P. (TRESCA) \\
\hline $55812.80021 \pm 0.00085$ & 447 & -0.00051 & Shadic S. (TRESCA) \\
\hline $55834.3372 \pm 0.0019$ & 454 & 0.00206 & Brát L. (TRESCA) \\
\hline $55837.4136 \pm 0.0025$ & 455 & 0.00211 & Trnka J. (TRESCA) \\
\hline $55840.48811 \pm 0.00063$ & 456 & 0.00028 & This work (CA $123 \mathrm{~cm})$ \\
\hline $55840.48845 \pm 0.00049$ & 456 & 0.00062 & This work (Loiano $152 \mathrm{~cm}$ ) \\
\hline $55886.6345 \pm 0.0021$ & 471 & 0.00148 & Dvorak S. (TRESCA) \\
\hline $56166.5776 \pm 0.0011$ & 562 & -0.00289 & This work (CA $220 \mathrm{~cm}$ BUSCA- $u$ ) \\
\hline $56166.58178 \pm 0.00057$ & 562 & 0.00129 & This work (CA $220 \mathrm{~cm}$ BUSCA-g) \\
\hline $56166.58162 \pm 0.00051$ & 562 & 0.00113 & This work (CA $220 \mathrm{~cm}$ BUSCA-r) \\
\hline $56166.57924 \pm 0.00054$ & 562 & -0.00124 & This work (CA $220 \mathrm{~cm}$ BUSCA-z) \\
\hline $56175.81123 \pm 0.00059$ & 565 & 0.00170 & Hose K. (TRESCA) \\
\hline $56203.49662 \pm 0.00031$ & 574 & -0.00002 & This work (Loiano $152 \mathrm{~cm}$ ) \\
\hline $56203.49682 \pm 0.00083$ & 574 & 0.00018 & This work (CA $123 \mathrm{~cm}$ ) \\
\hline
\end{tabular}

Notes. TRESCA and AXA refer to the "TRansiting ExoplanetS and CAndidates" and "Amateur Exoplanet Archive" websites, respectively.

without using stellar models, thus avoiding the dependence on stellar theory. These results are also given in Table 5.

Table 6 also contains a comparison between our own results and those found by Latham et al. (2009). We find smaller masses and radii for both the planet and the host star. This is surprising because the values of the fractional radius of the star, which furnishes the vital constraint on the stellar density, are very similar between the two studies (we find $r_{\mathrm{A}}=0.1590 \pm 0.0014$ versus $r_{\mathrm{A}}=0.1575_{-0.0089}^{+0.0041}$ for Latham et al. 2009). One small difference is that we adopted $T_{\text {eff }}=6130 \pm 80 \mathrm{~K}$ (Knutson et al. 2010) as opposed to the value of $6200 \pm 80 \mathrm{~K}$ used by Latham et al. (2009). Our revised physical properties move HAT-P-8 b into a region of parameter space which is comparatively well-supplied with transiting planets. Figure 4 shows the change in position in the planet mass-radius plot.

We contacted D. Latham in order to check the veracity of the final values of the parameters that they reported in their discovery paper. D. Latham confirmed that some of these results are indeed correct, and has kindly supplied revised values. These are from a re-analysis carried out by J. Hartman on a slight updated dataset, and are given in the final column of Table 6 . They correspond to a smaller planetary mass and radius $\left(M_{\mathrm{b}}=1.38 M_{\text {jup }}\right.$, $\left.R_{\mathrm{b}}=1.40 R_{\text {jup }}\right)$ than given in Latham et al. (2009), in agreement with our own findings.

\section{Variation of planetary radius with wavelength}

One of the factors that plays a principal role in determining the atmospheric properties of hot-Jupiter planets is the amount of stellar flux incident on the planet's surface. Variations in this irradiation cause different planets to have different atmospheric chemical mixing ratios and atmospheric opacities. This could potentially lead to divide into classes of hot Jupiters. An initial suggestion was that of Fortney et al. (2008) to distinguished pM- and pL-class planets, depending on the presence of strong absorbers such as gaseous titanium oxide ( $\mathrm{TiO})$ and vanadium oxide (VO) in their atmospheres. By observing a planetary transit at different wavelengths, it is possible to detect a variation in 
Table 5. Derived physical properties of the HAT-P-8 planetary system.

\begin{tabular}{|c|c|c|c|c|c|c|}
\hline & $\begin{array}{c}\text { This work } \\
\text { (dEB constraint) }\end{array}$ & $\begin{array}{c}\text { This work } \\
\text { (Claret models) }\end{array}$ & $\begin{array}{c}\text { This work } \\
\left(\mathrm{Y}^{2} \text { models }\right)\end{array}$ & $\begin{array}{c}\text { This work } \\
\text { (Teramo models) }\end{array}$ & $\begin{array}{c}\text { This work } \\
\text { (VRSS models) }\end{array}$ & $\begin{array}{c}\text { This work } \\
\text { (DSEP models) }\end{array}$ \\
\hline$K_{\mathrm{b}}\left(\mathrm{km} \mathrm{s}^{-1}\right)$ & $159.1 \pm 3.9$ & $154.8 \pm 2.3$ & $156.7 \pm 1.9$ & $153.4 \pm 1.7$ & $154.2 \pm 2.6$ & $155.4 \pm 2.13$ \\
\hline$M_{\mathrm{A}}\left(M_{\odot}\right)$ & $1.292 \pm 0.094$ & $1.189 \pm 0.054$ & $1.234 \pm 0.044$ & $1.158 \pm 0.037$ & $1.175 \pm 0.061$ & $1.202 \pm 0.053$ \\
\hline$R_{\mathrm{A}}\left(R_{\odot}\right)$ & $1.521 \pm 0.041$ & $1.480 \pm 0.032$ & $1.470 \pm 0.026$ & $1.467 \pm 0.023$ & $1.474 \pm 0.031$ & $1.485 \pm 0.026$ \\
\hline $\log g_{\mathrm{A}}(\mathrm{cgs})$ & $4.1852 \pm 0.0137$ & $4.1733 \pm 0.0088$ & $4.1954 \pm 0.0103$ & $4.1694 \pm 0.0105$ & $4.1715 \pm 0.0106$ & $4.1748 \pm 0.0115$ \\
\hline$M_{\mathrm{b}}\left(M_{\text {jup }}\right)$ & $1.345 \pm 0.071$ & $1.273 \pm 0.048$ & $1.305 \pm 0.041$ & $1.251 \pm 0.037$ & $1.263 \pm 0.053$ & $1.282 \pm 0.047$ \\
\hline$R_{\mathrm{b}}\left(R_{\text {jup }}\right)$ & $1.357 \pm 0.043$ & $1.320 \pm 0.034$ & $1.337 \pm 0.032$ & $1.308 \pm 0.030$ & $1.315 \pm 0.037$ & $1.325 \pm 0.034$ \\
\hline$\rho_{\mathrm{b}}\left(\rho_{\text {jup }}\right)$ & $0.503 \pm 0.034$ & $0.517 \pm 0.034$ & $0.511 \pm 0.033$ & $0.522 \pm 0.034$ & $0.519 \pm 0.035$ & $0.516 \pm 0.034$ \\
\hline$\Theta$ & $0.0691 \pm 0.0026$ & $0.0711 \pm 0.0023$ & $0.0702 \pm 0.0022$ & $0.0717 \pm 0.0022$ & $0.0714 \pm 0.0025$ & $0.0708 \pm 0.0023$ \\
\hline$a(\mathrm{AU})$ & $0.04510 \pm 0.00109$ & $0.04387 \pm 0.00066$ & $0.04442 \pm 0.00053$ & $0.04348 \pm 0.00047$ & $0.04370 \pm 0.00075$ & $0.04403 \pm 0.00065$ \\
\hline Age & - & $4.7_{-0.5}^{+1.4}$ & $3.5_{-0.7}^{+0.4}$ & $4.7_{-0.5}^{+1.0}$ & $4.2_{-0.4}^{+1.5}$ & $4.2_{-1.4}^{+0.5}$ \\
\hline
\end{tabular}

Table 6. Final physical properties of the HAT-P-8 system, compared with results from the literature.

\begin{tabular}{lcccc}
\hline \hline & This work (final) & Latham et al. (2009) & Moutou et al. (2011) & Latham (priv. comm.) \\
\hline$M_{\mathrm{A}}\left(M_{\odot}\right)$ & $1.192 \pm 0.061 \pm 0.043$ & $1.28 \pm 0.04$ & - & $1.28_{-0.06}^{+0.04}$ \\
$R_{\mathrm{A}}\left(R_{\odot}\right)$ & $1.475 \pm 0.032 \pm 0.010$ & $1.58_{-0.06}^{+0.08}$ & - & $1.57 \pm 0.07$ \\
$\log g_{\mathrm{A}}(\mathrm{cgs})$ & $4.177 \pm 0.011 \pm 0.019$ & $4.15 \pm 0.03$ & - & $4.15 \pm 0.03$ \\
$\rho_{\mathrm{A}}\left(\rho_{\odot}\right)$ & $0.371 \pm 0.013 \pm 0.018$ & - & - & $0.46 \pm 0.05$ \\
$M_{\mathrm{b}}\left(M_{\text {jup }}\right)$ & $1.275 \pm 0.053 \pm 0.030$ & $1.52_{-0.16}^{+0.18}$ & $1.34 \pm 0.05$ & $1.38 \pm 0.05$ \\
$R_{\mathrm{b}}\left(R_{\text {jup }}\right)$ & $1.321 \pm 0.037 \pm 0.016$ & $1.50_{-0.06}^{+0.08}$ & - & $1.40 \pm 0.08$ \\
$g_{\mathrm{b}}\left(\mathrm{ms}^{-1}\right)$ & $18.11 \pm 0.82$ & $16.98 \pm 1.17$ & - & $1.38 \pm 0.05$ \\
$\rho_{\mathrm{b}}\left(\rho_{\text {jup }}\right)$ & $0.517 \pm 0.035 \pm 0.006$ & $0.568 \pm 0048$ & - & $0.62 \pm 0.09$ \\
$T_{\mathrm{eq}}(\mathrm{K})$ & $1713 \pm 24 \pm 13$ & $1700 \pm 35$ & - & $1771 \pm 39$ \\
$\Theta$ & $0.0710 \pm 0.0025 \pm 0.0008$ & $0.061 \pm 0.003$ & - & $0.069 \pm 0.004$ \\
$a(\mathrm{AU})$ & $0.04390 \pm 0.00075 \pm 0.00052$ & $0.0487 \pm 0.0026$ & $0.0449 \pm 0.0007$ & $0.0450_{-0.0004}^{+0.0004}$ \\
Age $(\mathrm{Gyr})$ & $4.3_{-1.4-0.5}^{+1.5+0.8}$ & $3.4 \pm 1.0$ & - & $3.3_{-0.3}^{+0.7}$ \\
\hline
\end{tabular}

Notes. Where two errorbars are given, the first refers to the statistical uncertainties and the second to the systematic errors.

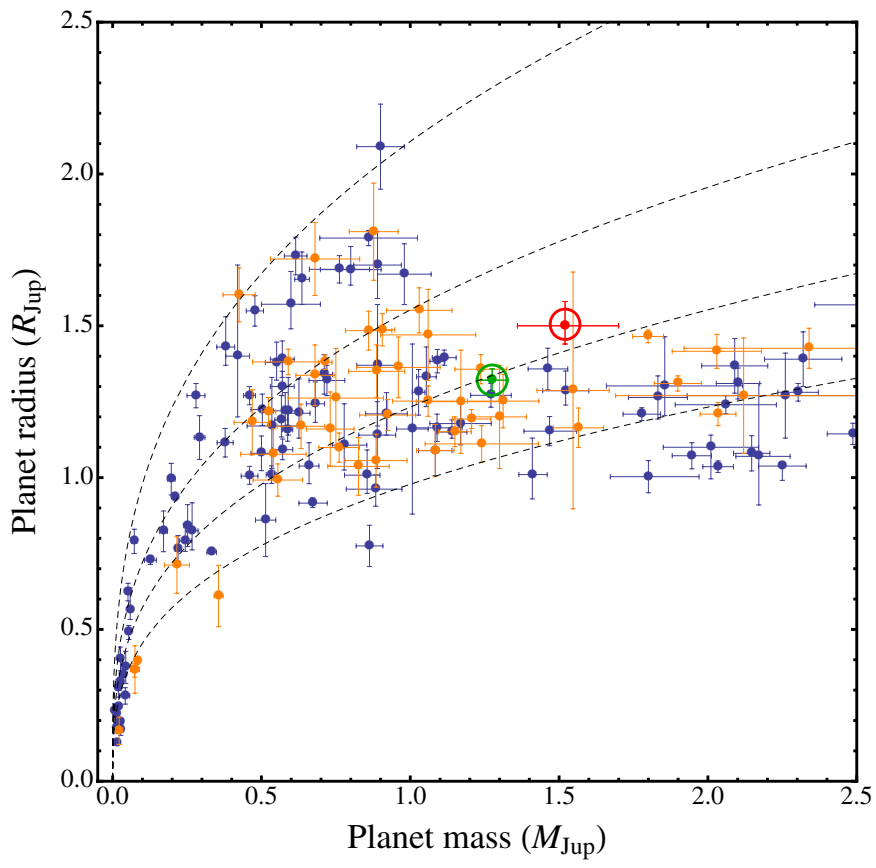

Fig. 4. Plot of the masses and radii of the known TEPs. The orange symbols denote values from the Homogeneous Studies project and the blue symbols results for the other known TEPs. HAT-P-8b is shown in red (Latham et al. 2009) and green (this work). Dotted lines show where density is (from bottom to top) $1.0,0.5,0.25$ and $0.1 \rho_{\text {Jup }}$.

the value of the radius measured as a function of the wavelength, and probe chemistry and wavelength dependent opacity at the planet's terminator. This dependence on the wavelength can be therefore used to probe the atmospheric composition of TEPs, being a complementary method to the observations of secondary eclipses.

As an additional possibility offered by the BUSCA data, we made an attempt to investigate possible variations of the radius of HAT-P-8 $b$ in different optical passbands. Receiving from its parent star an incident flux of $2.22 \pm 0.20 \times 10^{9} \mathrm{erg} \mathrm{s}^{-1} \mathrm{~cm}^{-2}$ (Latham, priv. comm.), HAT-P-8 b should belong to the $\mathrm{pM}$ class of planets. The theoretical models of Fortney et al. (2010) predict that its radius should be few percent lower at 350-400 nm and $800-950 \mathrm{~nm}$ versus $500-750 \mathrm{~nm}$. Following the strategy used by Southworth et al. (2012b), we fitted the BUSCA light curves with all parameters fixed to the final values reported in Table 4, with the exception of $k$ and the LD coefficients. The errors were estimated by a residual-permutation algorithm. The results are exhibited in Fig. 5, where the points show the data, the vertical bars represent the relative errors in the measurements and the horizontal bars show the full widths at half maximum transmission of the passbands used. As is apparent from Fig. 2, the BUSCA- $z$ light curve shows some structure in its residuals from phase 0 to 0.02 , which suggests that the $z$-band data suffer correlated noise. The result coming from this band should be therefore considered with caution. The optical region not covered by the BUSCA data was investigated by using the $i$-band light curve from Loiano, which is the best one that we have at this band (see Fig. 1).

A variation of $r_{\mathrm{b}}$ along the five passbands is clearly visible in Fig. 5. We investigated this variation with the help of model atmospheres. We used detailed non-grey atmosphere codes to model the temperature structure and transmission spectrum of 
the planet. We computed 1D model atmosphere of HAT-P-8 b, using the atmosphere code described in Fortney et al. (2005, 2008). Pressure-temperature profiles are derived that either include or exclude the opacity of TiO and VO molecules. The fully non-grey model uses the chemical equilibrium abundances of Lodders \& Fegley (2002) and the opacity database described in Freedman et al. (2008). The atmospheric pressure-temperature profiles simulate planet-wide average conditions or day-side average conditions. We computed the transmission spectrum of the models using the methods described in Fortney et al. (2010) and Shabram et al. (2011).

Excluding $\mathrm{TiO}$ and $\mathrm{VO}$ from the opacity calculation, in the upper panel of Fig. 5 we compare the transmissions spectrum of the 1D planet-wide average profile (in blue) with a warmer dayside average model (in green), to experimental data. In comparison, the bottom panel of Fig. 5 shows analogous calculations in red and yellow respectively, which include both the $\mathrm{TiO}$ and VO species. Coloured boxes indicate the predicted values for these models integrated over the bandpasses of the observations. Models without TiO and VO have optical transmission spectra that are dominated by Rayleigh scattering in the blue, and pressure-broadened neutral atomic lines of $\mathrm{Na}$ an $\mathrm{K}$ at $589 \mathrm{~nm}$ and $770 \mathrm{~nm}$, respectively. Models with $\mathrm{TiO}$ and VO absorption show significant optical absorption (a much large transit radius), that broadly peaks around $700 \mathrm{~nm}$, with a sharp falloff in the blue, and a shallower falloff in the red.

Comparing the panels of Fig. 5, it is readily apparent that the model that gives the best match to the data is the one with TiO and VO opacity in the atmosphere of HAT-P- $8 \mathrm{~b}$. The increased optical radius is somewhat larger than the model prediction. Taken at face value, the observations are in general agreement with the Fortney et al. (2008)'s hot-Jupiter classification based on stellar irradiation. We suggest that HAT-P-8 b should be an important target for followup studies to confirm or refute these suggestive observations. The clear detection of TiO/VO, or other strong optical absorbers, would be an important step in characterizing hot Jupiter atmospheres, as such absorbers are thought to cause temperature inversions in these atmospheres (e.g. Fortney et al. 2008; Burrows et al. 2008). Previously, Désert et al. (2008) suggested a detection of TiO and VO at strongly depleted levels in the red optical spectrum of HD 209845 b, using Hubble STIS.

\section{Light-curve anomalies}

While one of our follow-up light curves, obtained at the INT, displays a regular transit shape, the others all show anomalies of a similar shape. They display an asymmetry with respect the line of minimum transit time. It is important to clarify if the anomalies that we detected have an astrophysical nature or are of instrumental or environmental origin. One way to check this is to have independent measures of the same transit event, obtained using multiple telescopes located at different sites. In this way, if both the telescopes reported the same anomaly, it less likely that they are caused by instrumental or Earth-atmosphere effects. This already happened inadvertently in the case of several follow-up observations of WASP-33 carried out by amateur astronomers and reported in Kovács et al. (2012). It also occurred by chance for the transit observations of HAT-P-8 on the night of 2010/08/28, which was observed by ourselves at the INT and contemporaneously at CAHA by F. Hormuth. Figure 6 shows the two light curves in the same plot; we note that both have a regular transit shape but disagree over the transit depth. Some fraction of this disagreement is due to the different LD characteristics, as
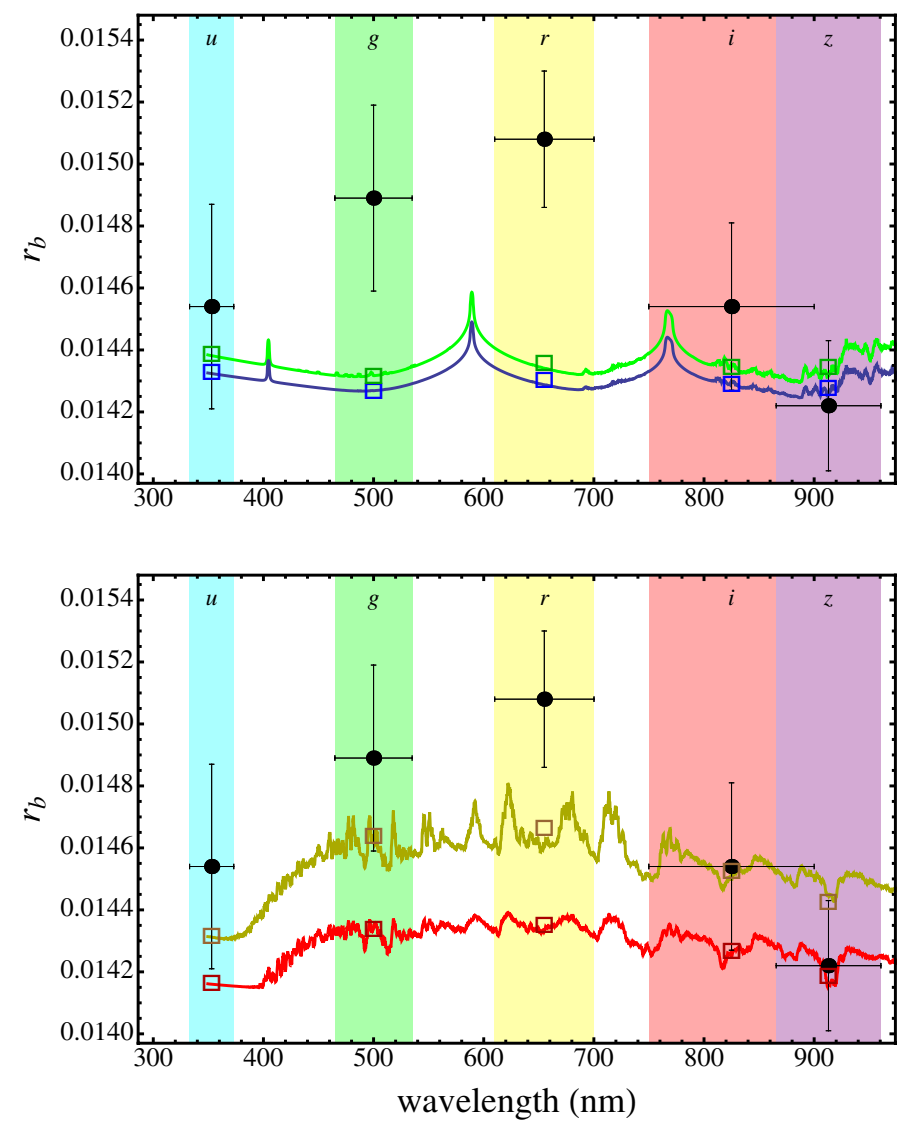

Fig. 5. Variation of the fractional planetary radius $r_{\mathrm{b}}=R_{\mathrm{b}} / a$ with wavelength. The points shown in the plot are from the Calar Alto $2.2 \mathrm{~m}(u$, $g, r$ and $z$ bands) and Loiano ( $i$-band) telescopes. The vertical bars represent the errors in the measurements and the horizontal bars show the full widths at half maximum transmission of the passbands used. The experimental points are compared with four models. These use planetwide average pressure-temperature profiles (in blue and red) or warmer day-side average profiles (in green and yellow). Synthetic spectra in the top panel do not include $\mathrm{TiO}$ and VO opacity, while spectra in the bottom do, based on equilibrium chemistry. Coloured squares represent band-averaged model radii over the bandpasses of the observations.

the INT data were obtained with a bluer filter than the CAHA data.

In 2011, we aimed to observe transits in the HAT-P-8 system from two different observatories and this goal was successfully achieved on the night of 2011/10/05 when a transit was simultaneously observed using the $1.52 \mathrm{~m}$ Cassini and CAHA $1.23 \mathrm{~m}$ telescopes. The datasets show partial but not complete agreement about the transit shape anomalies, as well as slight differences due to the different LD in the $i$ and $R$ passbands. Details of the two light curves are displayed in the lower panel of Fig. 7, while the upper panel shows another transit (2009, Loiano) which presents a similar anomaly. The anomalies cannot be removed by choosing different comparison stars for the differential photometry, but are not completely consistent between different datasets for the same transit. We have therefore treated them as correlated noise in our analysis (see Sect. 3).

If the recurrent anomalies that we noticed in the HAT-P-8 light curves are not of instrumental origin, is there a reasonable astrophysical explanation for these features? We can easily exclude a gravity darkening effect (e.g. Barnes 2009) because the rotation rate of the star is low (Latham et al. 2009; Moutou et al. 2011). The presence of a moon is an unlikely possibility, and in any case is difficult to constrain with so few observations. 


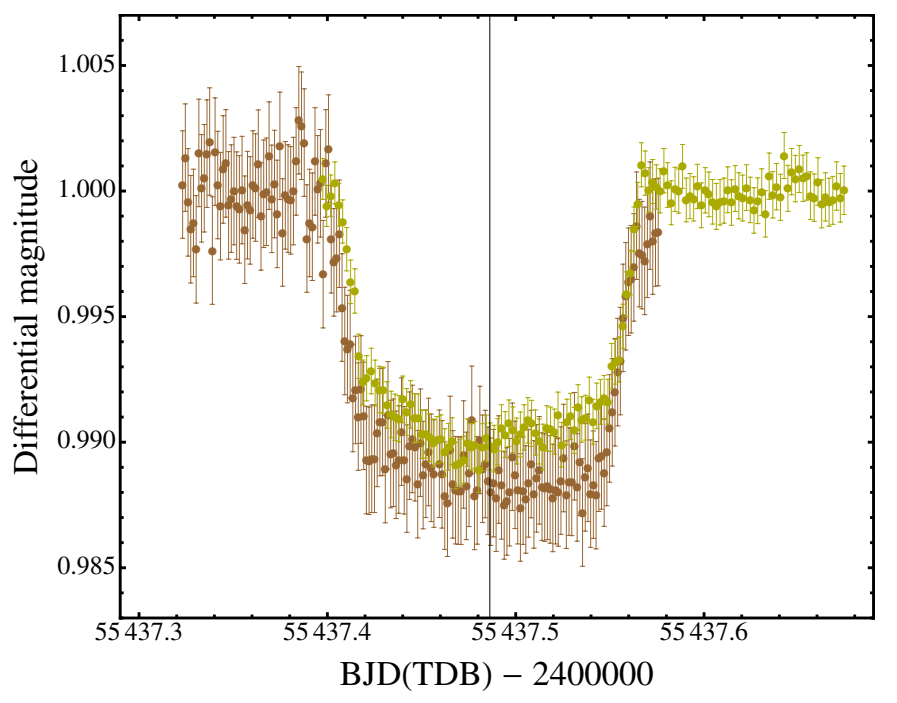

Fig. 6. Example of the consistency of follow-up observations made of the same transit on the night of $2010 / 08 / 28$ by different telescopes. This is an example of a "regular" transit. Lighter yellow dots are for the data taken at the INT (this work), whereas the darker brown ones for those taken by F. Hormuth (ETD) at the CAHA $1.23 \mathrm{~m}$. The agreement between the two datasets is not very good. The difference in depth between the two datasets is partly due to different filters used, Strömgren $y$ and Johnson $R$, respectively. The vertical line represents the expected transit minimum time.

Another possibility is that the anomalies are due to single or a belt of stellar spots on the photosphere of the parent star. Knutson et al. (2010) estimated that HAT-P-8 has an activity index of $\log \left(R_{H K}^{\prime}\right)=-4.985$, which indicates that the star has a moderate chromospheric activity. This is related to the star's magnetic structure and therefore to the presence of photospheric features, such as spots, that modulate luminosity. It is thus possible that, during the transit, dark starspots are occulted by the planet. Similar features were indeed already observed in several cases, e.g. HD 189733 (Pont et al. 2007), TrES-1 (Rabus et al. 2009), Kepler-17 (Désert 2011), HAT-P-11 (Sanchis-Ojeda \& Winn 2011), and WASP-19 (Tregloan-Reed et al. 2012). One argument against this is the relatively high $T_{\mathrm{eff}}$ of the star, $6130 \pm 80 \mathrm{~K}$, which makes starspots less likely to occur. Moreover, spots would only affect small parts of the light curve, while here both light curves show trends for the full duration of the transit.

Another possible explanation is that the faint M-dwarf companion of HAT-P-8 is a flare star, which could emit giant flares bright enough to significantly affect the light curves. However, the magnitude difference between the two objects $\left(\Delta i^{\prime}=7.34 \pm\right.$ 0.10 , Bergfors et al. 2012), is such that this star would have to generate a superflare in order to make a noticeable difference. One more explanation for this behaviour is that a significant fraction of the comparison stars display intrinsic variability which is insufficient to detect in individual stars but, when combined, is enough to modify the transit shape in the HAT-P-8 system.

Having exhausted all possibilities, we conclude that the apparent transit shape distortion shown in Fig. 7 could be caused by differential color extinction, pathological variability in the comparison stars, or other low-frequency noise of atmospheric or astrophysical origin. Further investigations of these hypotheses would, inevitably, require substantial further data and is beyond the scope of the current work.
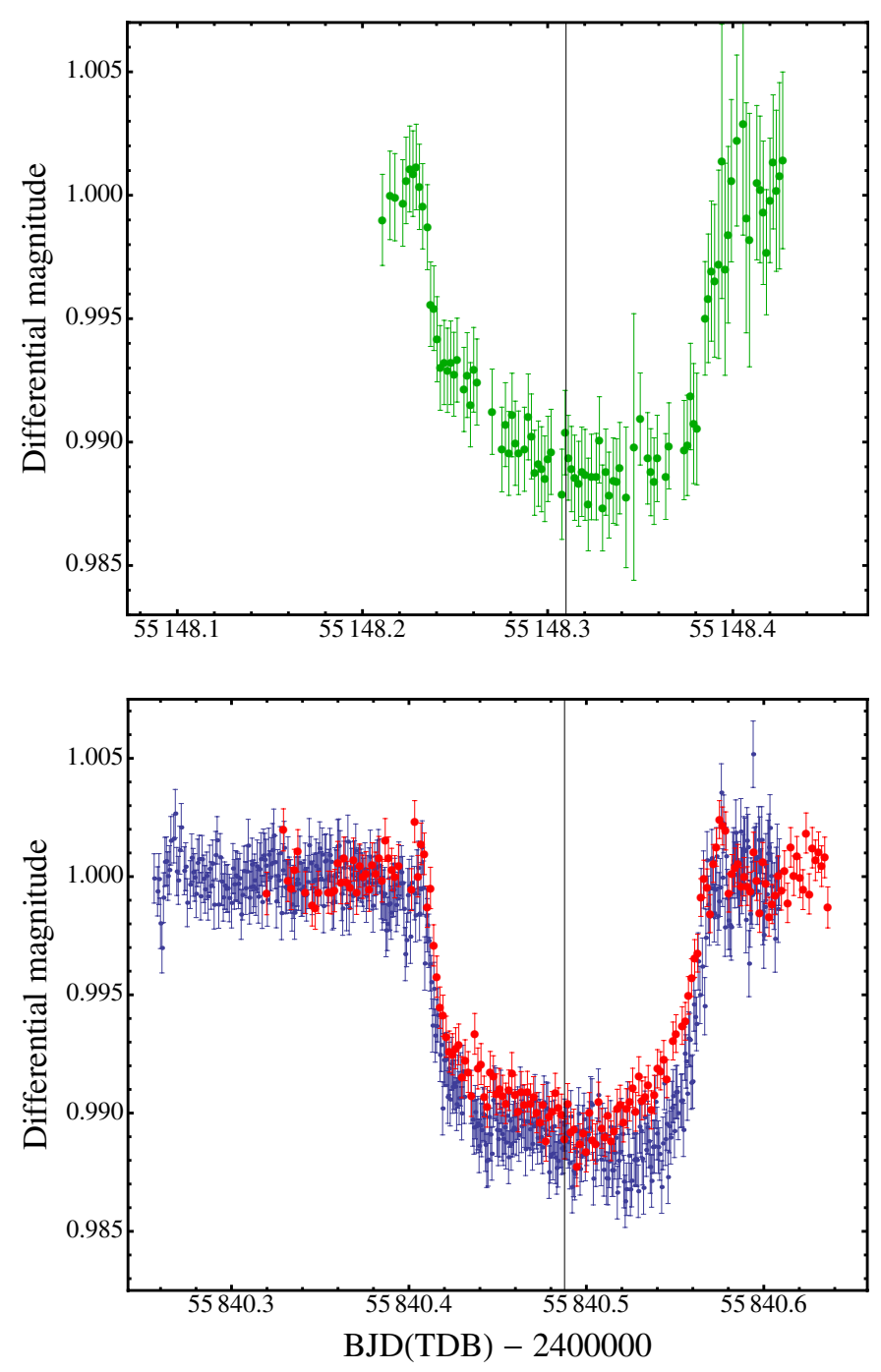

Fig. 7. Upper panel: an example of a discrepant transit. The green points are for the data taken at the Cassini telescope (this work) through a Gunn $r$ filter. Lower panel: an example of the consistency of followup observations made on the same date of 2011/10/05 by different observers. Lighter blue dots are for the data taken at the Cassini telescope (this work), whereas the red ones for those taken at CAHA $1.23 \mathrm{~m}$ (this work). Some of the difference in depth between the two datasets is due to different filters used, Gunn $i$ and Johnson $R$, respectively. The vertical line represents the expected transit minimum time.

\section{Summary and conclusions}

We have reported observations of six transits of the HAT-P-8 system performed using five different medium-class telescopes, for a total of eleven new light curves. All but one of these transits were obtained using the defocussed-photometry technique, achieving a photometric precision of $0.47-1.7$ mmag per observation. Four of them were observed on the same nights from two different telescopes. In one of these two nights, both the light curves show an anomaly which is probably caused by systematic noise of atmospheric or astrophysical origin. Another transit was simultaneously observed through four optical passbands in a wavelength range between 330 and $960 \mathrm{~nm}$.

We modelled our new and seven published datasets using the JKTEBOP code, and used the results to determine the physical properties of the planet and its host star. Compared to the discovery paper (Latham et al. 2009), we find a significantly smaller radius and mass for HAT-P- $8 \mathrm{~b}$. We obtain 
$R_{\mathrm{b}}=1.321 \pm 0.037 R_{\mathrm{Jup}}$ versus $1.50_{-0.06}^{+0.08} R_{\mathrm{Jup}}$, and $M_{\mathrm{b}}=1.275 \pm$ $0.053 M_{\text {Jup }}$ versus $1.52_{-0.16}^{+0.18} M_{\text {Jup }}$. The theoretical radius calculated by Fortney et al. (2007) for a core-free planet at age $4.5 \mathrm{Gyr}$ and distance $0.045 \mathrm{AU}$ is $1.107-1.108 R_{\mathrm{Jup}}$ for a planet of mass in the range $1.0-1.46 M_{\mathrm{Jup}}$, which is still significantly smaller than the radius we find.

Latham et al. (2009) found that HAT-P-8 b was a comparatively highly inflated planet. Instead, our results place it firmly in a well-populated part of the mass-radius diagram, removing its outlier status. Whilst the existing transit light curves of the HAT-P-8 system all show some systematic deviations from the best fits found using simple geometric models, the large number of available datasets means that our overall results are reliable. HAT-P-8 is another system where extensive follow-up photometry has been necessary to determine robust physical properties for a planetary system.

Finally, thanks to the ability of BUSCA to measure stellar flux simultaneously through different filters, covering a quite large range of optical window, we probed the composition of the atmosphere of HAT-P- 8 b by investigated how vary its radius in these wavelengths. In fact, the presence of strong optical absorbers in the atmosphere of the planet should produce larger transit radius at optical wavelengths than in the near UV or near infrared. We measured a variation of the radius of HAT-P-8 b along five passbands, corresponding to a wavelength coverage of 330-960 nm. This result was then theoretically investigated by using several synthetic spectra based on isothermal model atmospheres in chemical equilibrium. The comparison between the models and the experimental points suggests the presence of molecular gas that strongly absorbs in the optical, potentially composed of $\mathrm{TiO}$ and $\mathrm{VO}$ gases, in the atmosphere of HAT-P- $8 \mathrm{~b}$.

Acknowledgements. Based on observations collected at the Centro Astronómico Hispano Alemán (CAHA) at Calar Alto, Spain, operated jointly by the MaxPlanck Institut für Astronomie and the Instituto de Astrofísica de Andalucía (CSIC); observations obtained with the $1.52 \mathrm{~m}$ Cassini telescope at OAB Loiano Observatory, Italy; data collected at the Isaac Newton Telescope, operated on the island of La Palma, by the Isaac Newton Group in the Spanish Observatorio del Roque de los Muchachos. The reduced light curves presented in this work will be made available at the CDS (http://cdsweb.u-strasbg.fr/). We thank K. Todorov and R. F. Díaz for supplying photometric data. JS acknowledges financial support from STFC in the form of an Advanced Fellowship. We thank U. Thiele and R. Gualandi for their technical assistance at the CA $2.2 \mathrm{~m}$ telescope and Cassini telescope, respectively. We thank the anonymous referee for their useful criticisms and suggestions that helped us to improve the quality of the present paper. The following internet-based resources were used in research for this paper: the ESO Digitized Sky Survey; the NASA Astrophysics Data System; the SIMBAD data base operated at CDS, Strasbourg, France; and the arXiv scientific paper preprint service operated by Cornell University. LM thanks the HESS site in Namibia for the kind hospitality.

\section{References}

Ballester, G. E., Sing, D. K., \& Herbert, F. 2007, Nature, 445, 511 Barnes, J. W. 2009, ApJ, 705, 683
Bergfors, C., Brandner, W., Daemgen, S., et al. 2013, MNRAS, 428, 182 Burrows A., Budaj, J., \& Hubeny, I. 2008, ApJ, 678, 1436

Claret, A., 2004, A\&A, 428, 1001

Collier Cameron, A., Guenther, E., Smalley, B., \& Mcdonald, I. 2010, MNRAS, 407, 507

Désert, J.-M., Vidal-Madjar, A., Lecavelier Des Etangs, A., et al. 2008, A\&A, 492, 585

Désert, J.-M., Charbonneau, D., Demory, B.-O., et al. 2011, ApJS, 197, 14

Enoch, B., Collier Cameron, A., Parley, N. R., \& Hebb, L. 2010, A\&A, 516, A33

Fossati, L., Haswell, C. A., Froning, C. S., et al. 2010, ApJ, 717, L222

Fortney, J. J., Marley, M. S., Lodders, K., Saumon, D., \& Freedman, R. 2005, ApJ, 627, L69

Fortney, J. J., Marley, M. S., \& Barneset, J. W. 2007, ApJ, 659, 1661

Fortney, J. J., Lodders, K., Marley, M. S., \& Freedman, R. S. 2008, ApJ, 678, 1419

Fortney, J. J., Shabram, M., Showman, A. P., et al. 2010, ApJ, 709, 1396

Freedman, R. S., Marley, M. S., \& Lodders, K. 2008, ApJ, 174, 513

Gaudi, B. S., \& Winn, J. N. 2007, ApJ, 655, 550

Gazak, J. Z., Johnson, J. A., Tonry, J., et al. 2012, Adv. Astron., 2012, 697967

Gibson, N. P., Pollacco, D., Simpson, E. K., et al. 2008, A\&A, 492, 603

Gillon, M., Pont, F., Moutou, C., et al. 2006, A\&A, 459, 249

Hilditch, R. W. 2001, An Introduction to Close Binary Stars, (Cambridge, UK: Cambridge University Press)

Jones, B. W., \& Sleep, P. N. 2010, MNRAS, 407, 1259

Kipping, D. M., Fossey, S. J., \& Campanella, G. 2009, MNRAS, 400, 398

Knutson, H. A., Howard, A. W., \& Isaacson, H. 2010, ApJ, 720, 1569

Kovács, G., Kovács, T., Hartman, J. D., et al. 2012, A\&A, submitted

Latham, D. W., Bakos, G. A., Torres, G., et al. 2009, ApJ, 704, 1107

Leconte, J., Lai, D., \& Chabrier, G. 2011, A\&A, 528, A41

Li, S.-L., Miller, N. Lin, D. N. C., \& Fortney, J. J. 2010, Nature, 463, 1054

Lodders, K., \& Fegley, B. 2002, Icarus, 155, 393

Mancini, L., Nikolov, N., Southworth, J., et al. 2013, MNRAS, accepted [arXiv: 1301.3005]

Moutou, C., Díaz, R. F., Udry, S., et al. 2011, A\&A, 533, A113

Nikolov, N., Henning, Th., Koppenhoefer, J., et al. 2012, A\&A, 539, A159

Pont, F., Gilliland, R. L., Moutou, C., et al. 2007, A\&A, 476, 1347

Queloz, D., Eggenberger, A., Mayor, M., et al. 2000, A\&A, 359, L13

Rabus, M., Alonso, R., Belmonte, J. A, et al. 2009, A\&A, 494, 391

Sanchis-Ojeda, R., \& Winn, J. N. 2011, ApJ, 743, 61

Sanchis-Ojeda, R., Winn, J. N., Holman, M. J., et al. 2011, ApJ, 733, 127

Sanchis-Ojeda, R., Fabrycky, D. C., Winn, J. N., et al. 2012, Nature, 487, 449

Shabram, M., Fortney, J. J., Greene, T. P., \& Freedman, R. S. 2011, ApJ, 727, 65

Sing, D. K., Désert, J.-M., Lecavelier des Etangs, A., et al. 2009, A\&A, 505, 891

Simpson, E. K., Pollacco, D., Collier Cameron, A., et al. 2011, MNRAS, 414, 3023

Southworth, J. 2008, MNRAS, 386, 1644

Southworth, J. 2009, MNRAS, 394, 272

Southworth, J. 2010, MNRAS, 408, 1689

Southworth, J. 2011, MNRAS, 417, 2166

Southworth, J. 2012, MNRAS, 426, 1291

Southworth, J., Maxted, P. F. L., \& Smalley, B. 2004, MNRAS, 351, 1277

Southworth, J., Hinse, T. C., Jørgensen, U. G., et al. 2009, MNRAS, 396, 1023

Southworth, J., Bruni, I., Mancini, L., \& J. Gregorio 2012a, MNRAS, 420, 2580

Southworth, J., Mancini, L., Maxted, P. F. L., et al. 2012b, MNRAS, 422, 3099

Stetson, P. B. 1987, PASP, 99, 191

Swain, M. R., Vasisht, G., \& Tinetti, G. 2008, Nature, 452, 329

Szabó, Gy. M., Szabó, R., Benkõ, J. M., et al. 2011, ApJ, 736, L4

Todorov, K. O., Deming, D., Knutson, H. A., et al. 2012, ApJ, 746, 111

Torres, G., Winn, J. N., \& Holman, M. W. 2008, ApJ, 677, 1324

Tregloan-Reed, J., Southworth, J., \& Tappert, C. 2012, MNRAS, 428, 3671

Tusnski, L. R. M., \& Valio, A. 2011, ApJ, 743, 97

Winn, J. N., Holman, M. J., Torres, G., et al. 2008, ApJ, 683, 1076

Winn, J. N., Holman, M. J., Carter, J. A., et al. 2009, AJ, 137, 3826 